EPJ Web of Conferences 111, 10001 (2016)

DOI: $10.1051 /$ epjconf/201611110001

C) Owned by the authors, published by EDP Sciences, 2016

\title{
Dependence of Fission-Fragment Properties On Excitation En- ergy For Neutron-Rich Actinides
}

\author{
D. Ramos ${ }^{1, a}$, C. Rodríguez-Tajes ${ }^{1,2}$, M. Caamaño ${ }^{1}$, F. Farget ${ }^{2}$, L. Audouin ${ }^{3}$, J. Benlliure ${ }^{1}$, \\ E. Casarejos ${ }^{4}$, E. Clement $^{2}$, D. Cortina ${ }^{1}$, O. Delaune ${ }^{2, b}$, X. Derkx ${ }^{5}$, A. Dijon ${ }^{2}$, D. Doré ${ }^{6}$, \\ B. Fernández-Domínguez ${ }^{1}$, G. de France ${ }^{2}$, A. Heinz ${ }^{7}$, B. Jacquot ${ }^{2}$, A. Navin², C. Paradela ${ }^{2, c}$, \\ M. Rejmund ${ }^{2}$, T. Roger ${ }^{2}$, M.D. Salsac ${ }^{6}$, and C. Schmitt ${ }^{2}$ \\ ${ }^{1}$ GENP, USC, E-15784 Santiago de Compostela, Spain \\ ${ }^{2}$ GANIL, CEA/DMS-CNRS/IN2P3, BP 55027, F-14076 Caen Cedex 05, France \\ ${ }^{3}$ IPN Orsay, Université de paris-Sud, CNRS/IN2P3, F-91406 Orsay Cedex, France \\ ${ }^{4}$ CIMA, UVigo, E-36310 Vigo, Spain \\ ${ }^{5}$ LPC Caen, Université de Caen Basse-Normandie-ENSICAEN-CNRS/IN2P3, F-14050 Caen Cedex, France \\ ${ }^{6}$ CEA Saclay, DMS/IRFU/SPhN, 91191 Gif-sur-Yvette Cedex, France \\ ${ }^{7}$ Chalmes University of Technology, SE-41296 Göteborg, Sweden
}

\begin{abstract}
Experimental access to full isotopic fragment distributions is very important to determine the features of the fission process. However, the isotopic identification of fission fragments has been, in the past, partial and scarce. A solution based on the use of inverse kinematics to study transfer-induced fission of exotic actinides was carried out at GANIL, resulting in the first experiment accessing the full identification of a collection of fissioning systems and their corresponding fission fragment distribution. In these experiments, $\mathrm{a}^{238} \mathrm{U}$ beam at $6.14 \mathrm{AMeV}$ impinged on a carbon target to produce fissioning systems from $\mathrm{U}$ to Am by transfer reactions, and $\mathrm{Cf}$ by fusion reactions.

Isotopic fission yields of ${ }^{250} \mathrm{Cf},{ }^{244} \mathrm{Cm},{ }^{240} \mathrm{Pu},{ }^{239} \mathrm{~Np}$ and ${ }^{238} \mathrm{U}$ are presented in this work. With this information, the average number of neutrons as a function of the atomic number of the fragments is calculated, which reflects the impact of nuclear structure around $\mathrm{Z}=50$, $\mathrm{N}=80$ on the production of fission fragments. The characteristics of the Super Long, Standard I, Standard II, and Standard III fission channels were extracted from fits of the fragment yields for different ranges of excitation energy. The position and contribution of the fission channels as function of excitation energy are presented.
\end{abstract}

\section{Introduction}

The fission of the nucleus is a complex process where intrinsic excitation energy transforms into a large deformation until the nucleus splits. During this collective motion, the single-particle shell structure of nucleons strongly influences the process if the excitation energy of the fissioning system remains moderate [1-3].

\footnotetext{
a e-mail: diego.ramos@usc.es

${ }^{b}$ Present address: CEA DAM DIF, F-91297 Arpajon, France

${ }^{\mathrm{c}}$ Present address: EC-JRC-IRMM, Retieseweg 111, B-2440 Geel, Belgium
} 
In general, experimental observations are limited to few observables, making it difficult to isolate the impact of specific nuclear properties on the whole process [4-6]. The present work, based on the use of inverse kinematics, provides a new set of measurements that relates the excitation energy of the fissioning system, produced either by fusion or transfer reactions, to the characteristics of the full isotopic fragment distribution. These new measurements for fissioning systems, some of them not accessible with any other technique, also provide nuclear data, important for nuclear energy applications, such as the development of next-generation nuclear reactors or the recycling of radioactive waste.

\section{Experimental Setup}

A ${ }^{238} \mathrm{U}$ beam, accelerated to $6.14 \mathrm{~A} \mathrm{MeV}$ of energy, impinges on a $100 \mu \mathrm{g} / \mathrm{cm}^{2}{ }^{12} \mathrm{C}$ target. Transfer reactions take place producing n-rich actinides with a certain excitation-energy distribution. The target-like nucleus is detected by using a telescope composed of two double-sided, annular, Si detectors. The two elements of the telescope $(\Delta \mathrm{E}, \mathrm{E})$ provide the isotopic identification. The segmentation of the detector permits the scattering angle to be measured, which, together with the measurement of the energy, allows for the reconstruction of the excitation energy [7]. A fusion reaction is assumed for every fission event that is registered without any target-like recoil detected in the telescope.

The corresponding actinide may decay by fission. In such a case, one of the fragments passes through the VAMOS spectrometer and is fully identified in terms of charge state, atomic, and mass number at the focal plane. The detection setup includes a multiwire chamber for time-of-flight measurements, two drift chambers for position and angle determination, and an ionization chamber and a wall of Si detectors to perform dE-E measurements. The angles at the target position and the energy of the fission fragments are reconstructed by back-tracing methods based on the ionic optics of the spectrometer [8-10]. Finally, six clovers of the EXOGAM array of Ge detectors were surrounding the target for $\gamma$-ray measurements, yielding information on the sharing of the excitation energy between the fissioning system and the target-like recoil [7], and providing a cross-check of the fragment identification [11].

\section{Fission Products}

Data on five different fissioning systems are reported in this work: ${ }^{250} \mathrm{Cf}$, produced in fusion reactions with a well-defined excitation energy of $46 \mathrm{MeV},{ }^{244} \mathrm{Cm},{ }^{240} \mathrm{Pu},{ }^{239} \mathrm{~Np}$, and ${ }^{238} \mathrm{U}$, produced in transfer reactions with distributions of excitation energy centered at $23 \mathrm{MeV}, 10.7 \mathrm{MeV}, 7.5 \mathrm{MeV}, 7.4 \mathrm{MeV}$ respectively, with a full width at half maximum of approximately $8 \mathrm{MeV}$ [7]. Figure 1 shows the excitation energy distributions for each fissioning system produced by transfer reactions. In the case of ${ }^{238} \mathrm{U}$, a $23 \%$ contamination from random coincidences between elastic scattering with fusion-fission events was subtracted. Figure 2 (a) shows the fission yields of these systems as a function of the fragment atomic number. Lighter fissioning systems at lower excitation energy show mostly asymmetric fission -this is the case of ${ }^{238} \mathrm{U},{ }^{239} \mathrm{~Np}$, and ${ }^{240} \mathrm{Pu}$ - while symmetric fission becomes more relevant for heavier fissioning systems at higher excitation energy, such as ${ }^{244} \mathrm{Cm}$ and ${ }^{250} \mathrm{Cf}$. In the asymmetric fission, the heavier group of the distribution is observed to remain in the same Z-region for the different fissioning systems while the lighter group moves with the fissioning system. This behavior reflects the presence of structure effects at scission point, making certain configurations more favored than others, independently of the fissioning system. Figure 2 (b) shows that the stabilization of the heavy group is also present when displaying the fission yields as a function of the mass of the post-neutron evaporation fission fragments for the same fissioning systems. Figure 2 (c) shows the neutron excess, 
defined as the ratio of the average neutron number over the atomic number, of the post-neutron evaporation fission fragments. The observed sawtooth behavior is the signature of shell effects driven of $<\mathrm{N}>\approx 80$. This behavior becomes smoother for the systems with higher excitation energy, which is coherent with the damping of shell effects. The sawtooth behavior of the neutron excess was already observed to evolve with the excitation energy for the case of ${ }^{240} \mathrm{Pu}[12]$.
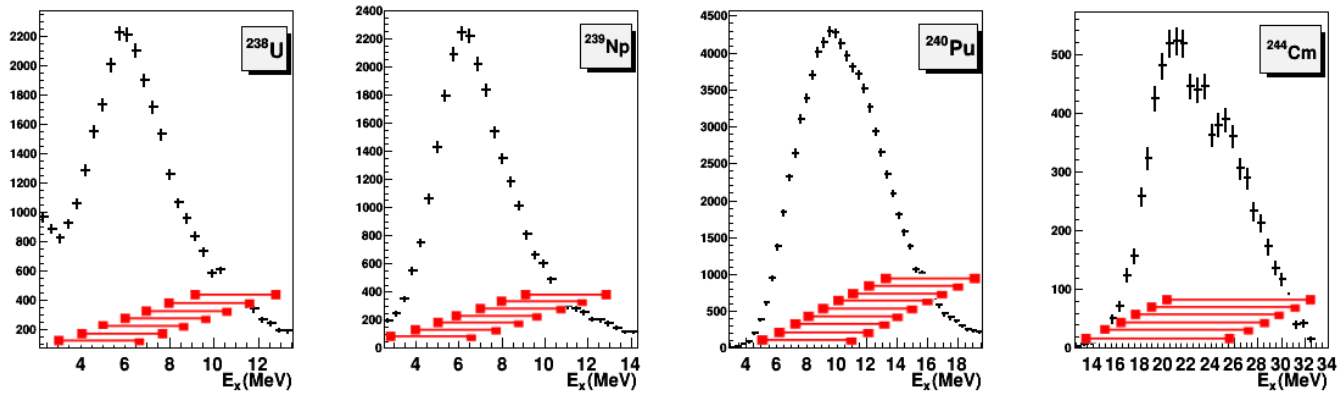

Figure 1. Distribution on excitation energy for each fissioning system produced by transfer reactions. Red lines indicates the gates on excitation energy considered for investigate the evolution of the fission-fragment distribution with the excitation energy.

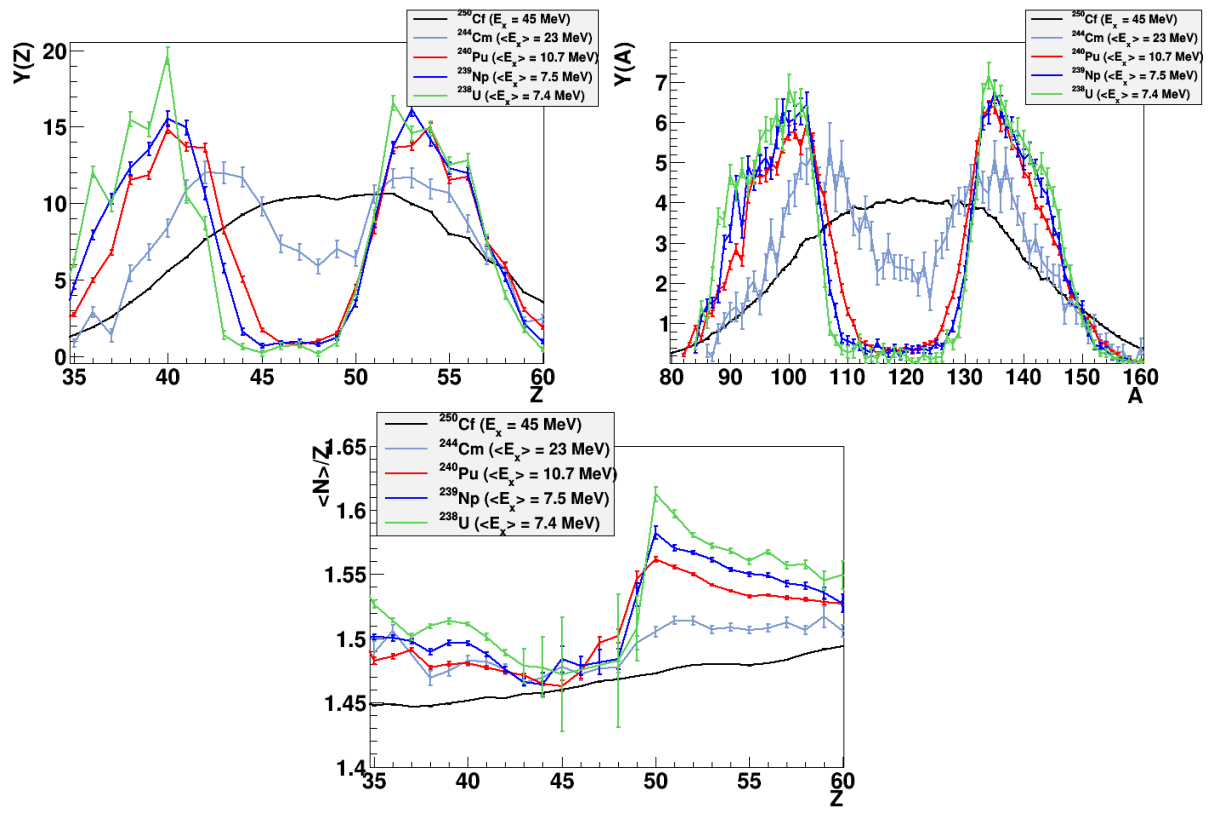

Figure 2. (a) Atomic number distributions of fission fragments for different fissioning systems. (b) Mass distributions of post-neutron evaporation fission fragments. (c) Neutron excess of post-neutron evaporation fission fragments as a function of their atomic number. 

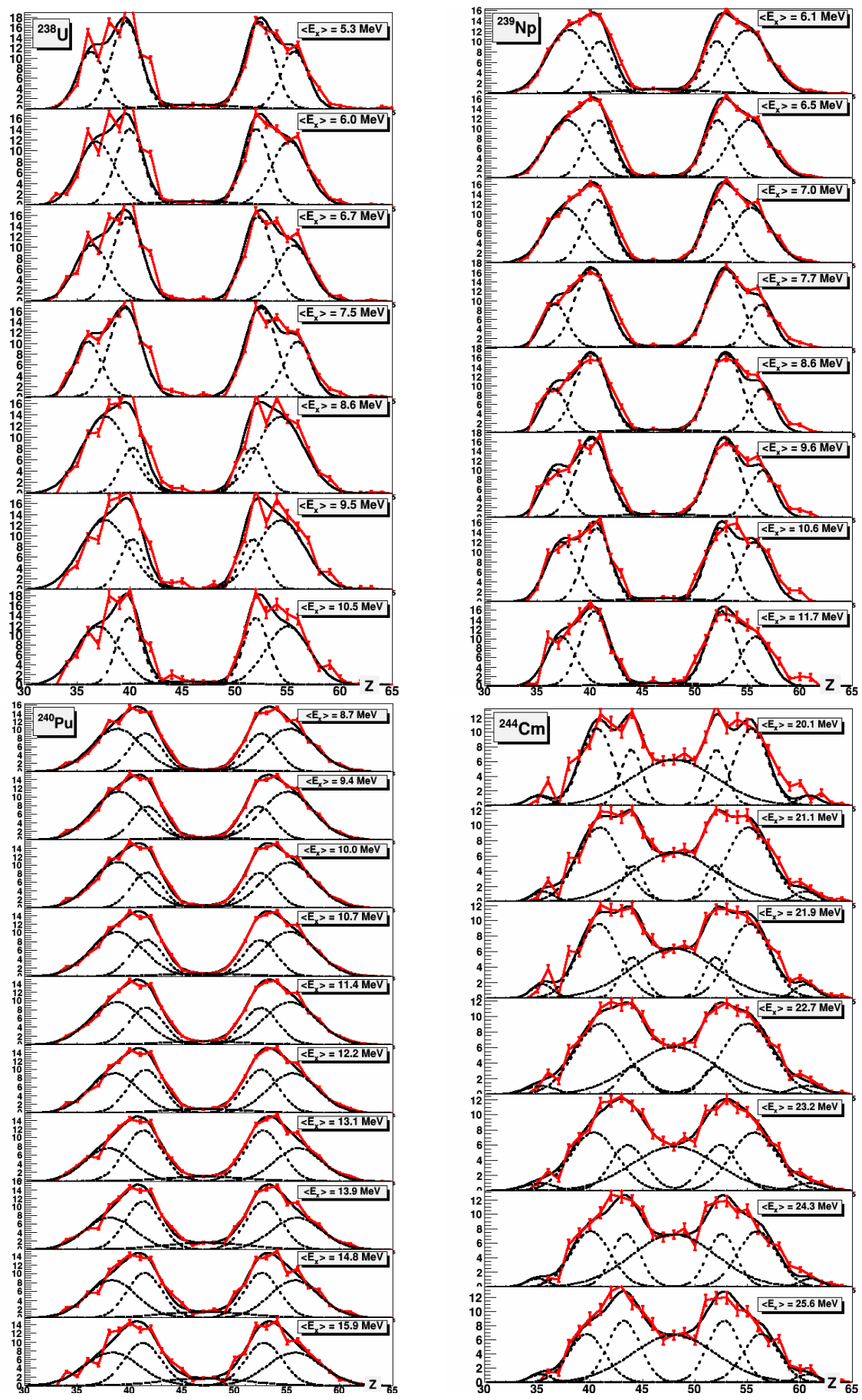

Figure 3. Atomic number distribution of fragments produced in the fission of ${ }^{238} \mathrm{U},{ }^{239} \mathrm{~Np},{ }^{240} \mathrm{Pu}$ and ${ }^{244} \mathrm{Cm}$. Different ranges of excitation energy were investigated. The Standard I and Standard II fission channels are fitted to the distributions of ${ }^{238} \mathrm{U},{ }^{239} \mathrm{~Np}$ and ${ }^{240} \mathrm{Pu}$. In the case of ${ }^{244} \mathrm{Cm}$, the Standard III channel was needed to be included. The Super Long channel is fixed, the amplitude corresponds to the value at the distribution in the symmetry, and its width is fixed to 4 , following the prescription [13]. 

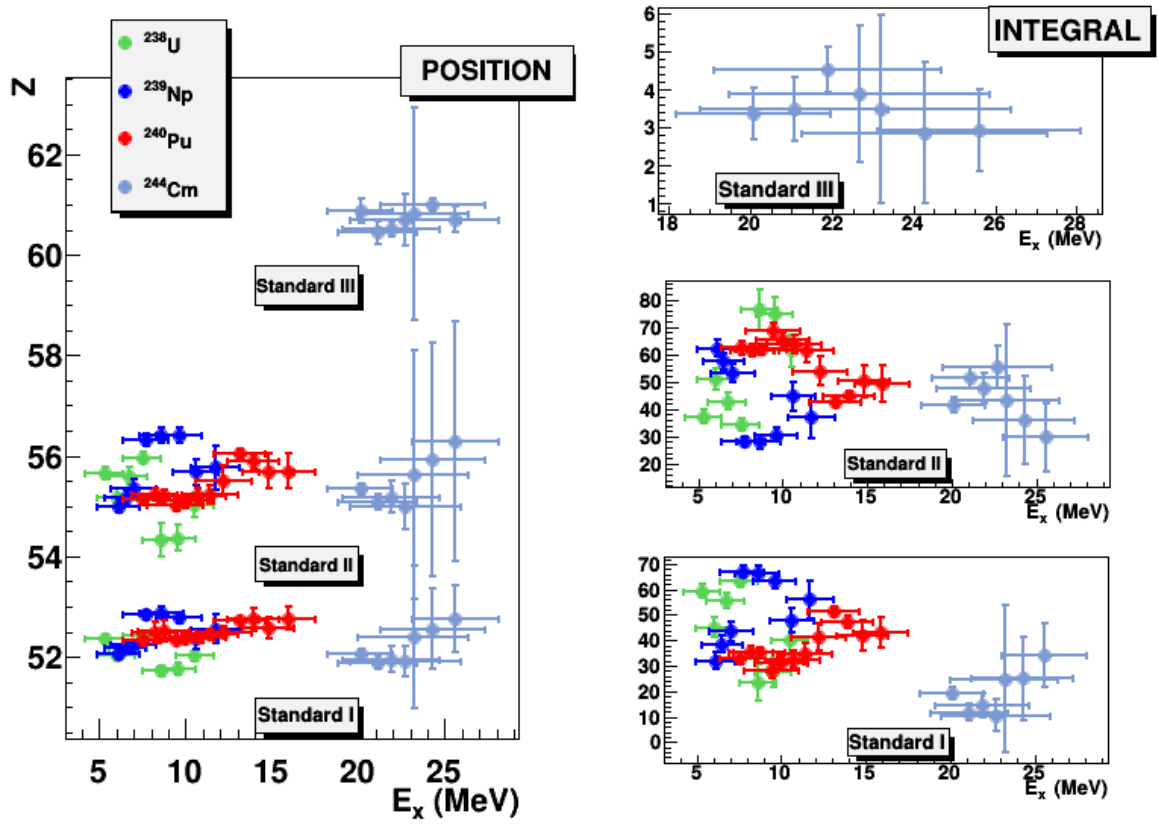

Figure 4. (left)Fitted positions and (right) integrals of the Standard I, Standard II and Standard III fission channels for ${ }^{238} \mathrm{U},{ }^{239} \mathrm{~Np},{ }^{240} \mathrm{Pu}$ and ${ }^{244} \mathrm{Cm}$ as function of the excitation energy. A general trend in the position of the channels with the excitation energy is observed.

The fission fragment distribution is investigated also by scanning the excitation energy induced in the transfer reaction [7]. Overlapping gates are defined in the excitation energy distribution for the fissioning systems produced by transfer reactions; these gates are shown in Figure 1. The atomic number fragment distribution is studied for each gate and each fissioning system. Figure 3 shows the $\mathrm{Z}$ distributions of fission fragments for different ranges of excitation energy in the case of (a) ${ }^{238} \mathrm{U}$, (b) ${ }^{239} \mathrm{~Np}$, (c) ${ }^{240} \mathrm{Pu}$, and (d) ${ }^{244} \mathrm{Cm}$. Also presented, is a fit of these distributions with different fission channels. The Super Long, Standard I, and Standard II fission channels are considered for the lightest systems, namely ${ }^{238} \mathrm{U},{ }^{239} \mathrm{~Np}$, and ${ }^{240} \mathrm{Pu}$. For the heavier system, ${ }^{244} \mathrm{Cm}$, the Standard III channel has to be included to describe the distribution. The parameters of the Super Long channel are fixed: the amplitude corresponds to the value of the distribution at the symmetry position and the width is fixed to 4, following the prescription given in Ref. [13]. Figure 4 shows the positions (left) and the integrals (right) of the Standard I, Standard II, and Standard III fission channels obtained from the Z distribution fits as a function of the excitation energy. An evolution of the positions of the asymmetric fission channels is observed with the excitation energy. In general, there is an increase of the position with the excitation energy except in the case of ${ }^{238} \mathrm{U}$, which shows a fast change with the excitation energy that can be explained by a remaining contamination from ${ }^{250} \mathrm{Cf}$ at low excitation energy. In Figure 5, the Super Long, Standard I, Standard II, and Standard III fission channels are fitted to the fragment $Z$ distribution from fission of ${ }^{250} \mathrm{Cf}$ where only one well-defined excitation energy of $46 \mathrm{MeV}$ is available. The positions of the S I, S II, and S III were fitted to 53.0, 56.1, and 59.7 respectively, the integrals were fitted to $5.2,27.2$, and 16.8 respectively. 


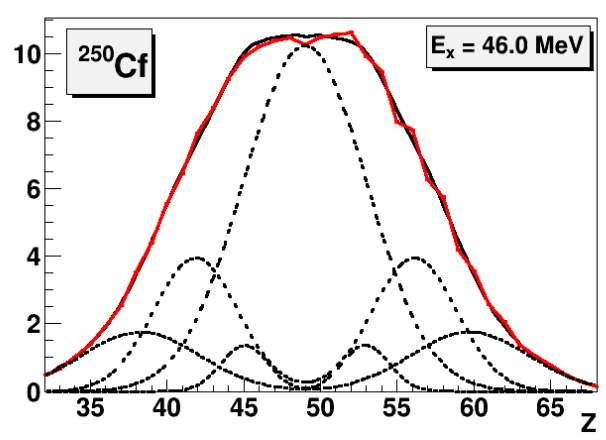

Figure 5. Atomic number distribution of fragments produced in the fission of ${ }^{250} \mathrm{Cf}$ with a well-defined excitation energy of $46 \mathrm{MeV}$. The Standard I, Standard II and Standard III fission channels are fitted to the distributions. The Super Long channel is fixed; the amplitude corresponds to the value of the distribution at symmetry, and its width is fixed to 4, following the K.-H. Schmidt prescription [13] . The positions of the S I, S II and S III were fitted to $53.0,56.1$ and 59.7, respectively, the integrals were fitted to 5.2, 27.2 and 16.8, respectively.

\section{Conclusions and outlook}

This experiment allowed the study of fission of five different systems, ${ }^{238} \mathrm{U},{ }^{239} \mathrm{~Np},{ }^{240} \mathrm{Pu},{ }^{244} \mathrm{Cm}$, and ${ }^{250} \mathrm{Cf}$, most of them exotic nuclei. The reconstruction of the fission-induced reactions and the isotopic identification of their fission products show the evolution of the characteristics of the fragments as a function of the available excitation energy of the fissioning system.

The contribution of symmetric fission is observed to increase for heavier fissioning systems with higher excitation energy, as expected. The investigation of the neutron excess of the fragments reflects nuclear-structure effects driven of $\langle\mathrm{N}>\approx 80$ in the region of $\mathrm{Z} \approx 50$ that increase for lower excitation energies and lighter fissioning systems.

The study of the fission channels shows an evolution of the positions of the asymmetric channels, Standard I, Standard II, and Standard III with the excitation energy.

Finally, fragment velocities and total kinetic energies will become available as the analysis progresses and a more precise parametrization of the fission channels, taking into account the total kinetic energy distribution of the fission fragments, will be performed.

This work was partially supported by the Spanish Ministry of Research and Innovation under the Grants No. FPA2010-22174-C02-01 and RYC-2012-11585.

\section{References}

[1] J. L. Sida et al., Nucl. Phys. A 502, 233c (1989).

[2] S. Steinhäuser et al., Nucl. Phys. A 634, 89 (1998).

[3] C. Böckstiegel et al., Nucl. Phys. A 802, 12 (2008).

[4] A. Bail et al., Phys. Rev. C 84, 034605 (2011).

[5] J.-P. Bocquet and R. Brissot, Nucl. Phys. A 502, 213 (1989).

[6] W. Lang et al., Nucl. Phys. A 345, 34 (1980).

[7] C. Rodríguez-Tajes et al., Phys. Rev. C 89, 24614 (2014).

[8] S. Pullanhiotan et al., Nucl. Instr. and Methods A 593, 343 (2008). 
[9] M. Rejmund et al., Nucl. Instr. and Methods A 646, 184 (2011).

[10] M. Caamaño et al., Phys. Rev. C 88, 24605 (2013).

[11] A. Shrivastava et al., Phys. Rev. C 80, 051305 (2009).

[12] D. Ramos et al., Acta Phys. Pol. B 46, 443 (2015).

[13] K.-H. Schmidt et al., Nucl. Phys. A 665, 221 (2000). 
